stoffatomen unter Aufnahme von Natriumbisulfit gelöst werden kann. Es ist das z. B. nachgewiesen worden für die Zimmtsäure ${ }^{20}$ ), die Fumar - und Maleïnsänre ${ }^{21}$ ), das Styrol ${ }^{22}$ ), das Mesityloxyd und Phoron ${ }^{23}$ ) n. a. m. Aehnlich könnten die Verhältnisse beim Dihydrocarvon liegen. Versuche über diesen Punkt sind im Gange. Andere Beobachtungen, welche über die Art der Atombindung in den hier besprochenen Körpern Auskunft zu geben versprechen, werden zum Theil schon in der folgenden Abhandlung mitgetheilt werden.

Ueber die beiden isomeren Benzylderivate des Nitroso- $\beta$-benzylhydroxylamins; von Rudolf Lindner.

Aus dem I. ehemischen Laboratorium der Unjversität Leipzig; (mitgetheilt von Robert Behrend). (Eingelanfen am 22. März 1893.)

Nach den Versuchen von Behrend und $\mathrm{K}_{0} \mathrm{nig}^{1}$ ) wird $\beta$-Benzylhydroxylamin durch salpetrige Säure in ein Nitrosoderivat übergeführt, dessen Natriumsalz beim Erwärmen mit Benzylchlorid in alkoholischer Lösung einen bei $58-59^{\circ}$ schmelzenden Benzylester liefert. Nimmt man an, dass das Nitroso- $\beta$-benzylhydroxylamin die Constitution eines Nitrosamins besitzt, so ist zu erwarten, dass sein Benzylester mit dem Nitrosamin des $\alpha$-Dibenzylhydroxylamins identisch ist:

$$
\begin{aligned}
& \mathrm{C}_{7} \mathrm{H}_{7} \mathrm{~N}(\mathrm{NO}) \mathrm{ONa}+\mathrm{C}_{7} \mathrm{H}_{7} \mathrm{Cl}=\mathrm{C}_{7} \mathrm{H}_{7} \mathrm{~N}(\mathrm{NO}) \mathrm{OC}_{7} \mathrm{H}_{7}+\mathrm{NaCl} \\
& \mathrm{C}_{7} \mathrm{H}_{7} \mathrm{NHOC}_{7} \mathrm{H}_{7}+\mathrm{HNO}_{2}=\mathrm{C}_{7} \mathrm{H}_{7} \mathrm{~N}(\mathrm{NO}) \mathrm{OC}_{7} \mathrm{H}_{7}+\mathrm{H}_{9} \mathrm{O} .
\end{aligned}
$$

${ }^{20)}$ Diese Annalen 154, 63 .

21) Diesc Annalen 157, 15.

22) Jahresbericht $1875,384$.

23) Ber. d. deutsch. chem. Ges. 15, 592:

1) Diese Annalen $\mathbf{2 6 3}, 217$. 


\section{Lindner, Ueber die beiden isomeren Benzylderivate}

Nun ist aber das Nitroso- $\alpha$-dibenzylhydroxylamin völlig verschieden vou dem Benzylester des Nitroso- $\beta$-benzylhydroxylamins. Es krystallisirt in Nadeln vom Schmelzpunkt $73-74^{\circ},{ }^{2}$ ) während der letztere Körper Blättchen vom Schmelzpunkt $58-59^{\circ}$ bildet.

Beide Körper besitzen dieselbe Zusammensetzung, das gleiche Molekulargewicht, beide sind unlöslich in Alkalien und zeigen die Liebermann'sche Nitrosoreaction. Unterschieden sind sie ausser durch ihre physikalischen Eigenschaften durch ihre sehr ungleiche Widerstandsfähigkeit gegen die Einwirkung von Säuren, Alkalien und Reductionsmitteln, und zwar ist die bei $59^{\circ}$ schmelzende Verbindung weitaus die unbeständigere von beiden. Tritt aber die Reaction ein, so liefern beide Körper dieselben Producte. Entweder besitzt also das Nitroso- $\beta$-benzylhydroxylamin oder das Nitroso- $\alpha$-dibenzylhydroxylamin nicht die Constitution eines Nitrosamins, womit aber alle ihre Eigenschaften in Einklang stehen, oder bei der Benzylirung des Nitroso- $\beta$-benzylhydroxylamins tritt Umlagerung ein oder endlich handelt es sich um einen Fall von Stereoisomerie.

Von Wichtigkeit für die Beurtheilung der Frage ist die Thatsache, dass bei der Benzylirung des Nitroso- $\beta$-benzylhydroxylamins neben dem bei $59^{\circ}$ schmelzenden Ester, wenn auch in untergeordneter Menge, die bei $74^{0}$ schmelzenden Nadeln des Nitroso- $\alpha$-dibenzylhydroxylamins entstehen. Vermuthlich bilden sich dieselben ursprünglich in viel grösserer Menge, ja sie stellen wohl gar das Hauptproduct der Reaction dar, da neben $30-40$ pC. der theoretischen Ausbeute an Blättchen und geringen Mengen der Nadeln bedeutende Mengen öliger Producte auftreten. Es wird aber gezeigt werden, dass die Nadeln beim Erwärmen ihrer alkoholischen Lösung bei Gegenwart von Natriumäthylat sehr schnell in ölige Verbindungen verwandelt werden, während sowohl die Blättchen als auch das Nitroso- $\beta$-benzylhydroxylamin unter diesen Umständen sehr beständig sind.

श) a. a. $0, \mathrm{~S}, 220$. 
Man wird demnach annehmen dürfen, dass das Nitroso$\beta$-benzylbydroxylamin die Constitution eines wahren Nitrosamins besitzt und als normales Product der Benzylirung das Nitrosamin des $\alpha$-Dibenzylhydroxylamins liefert. Die Blättchen dagegen dürften, wie nach Mittheilung der Versuchsergebnisse begründet werden wird, einer Umlagerung ihre Entstehung verdanken.

\section{Darstellung der isomeren Verbindungen.}

Das Nitroso- $\beta$-benzylhydroxylamin wurde auf die von Behrend and König angegebene Weise dargestellt.

Bringt man die Verbindung in alkoholischer Lösung mit der äquivalenten Menge Natriumäthylat zusammen, so scheidet sich das Natriumsalz als Krystallbrei ab, der nach dem Waschen mit Alkohol und Aether analysenrein ist. Das Salz schmilzt bei $233-234^{\circ}$ unter Zersetzung.

$0,1383 \mathrm{~g}$ gaben $0,0 \check{68} \mathrm{Na}_{2} \mathrm{SO}_{4}$.

$\begin{array}{ccc} & \text { Berechnet für } & \text { Gefunden } \\ & \mathrm{C}_{7} \mathrm{H}_{7} \mathrm{O}_{2} \mathrm{~N}_{2} \mathrm{Na} & \\ \mathrm{Na} & 13,22 & 13,30\end{array}$

Das Silbersalz fällt beim Versetzen der wässrigen Lösung des Natriumsalzes mit Silbernitrat als voluminöser Niederschlag, der bei $108-109^{\circ}$ unter Zersetzung schmilzt.

$0,2012 \mathrm{~g}$ gaben $0,1110 \mathrm{AgCl}$.

$\begin{array}{ccc} & \text { Berechnet für } & \text { Gefunden } \\ & \mathrm{C}_{7} \mathrm{H}_{7} \mathrm{O}_{2} \mathrm{~N}_{2} \mathrm{Ag} & \\ \mathrm{Ag} & \mathbf{4 1 , 7 0} & \mathbf{4 1 , 5 2}\end{array}$

Der bei $58-59^{\circ}$ schmelzende Benzylester wurde ebenfalls genan nach den Angaben von Behrend und König dargestellt. Die höchste Ausbeute betrug etwa $43 \mathrm{pC}$. der berechneten.

$0,2850 \mathrm{~g}$ gaben $0,7230 \mathrm{CO}_{2}$ und $0,1470 \mathrm{H}_{2} \mathrm{O}$.

$0,2537 \mathrm{~g} \# \quad 0,6429 \mathrm{CO}_{2} \leadsto 0,1399 \mathrm{H}_{2} \mathrm{O}$.

$0,2686 \mathrm{~g} " 27,20 \mathrm{ccm}$ Stickgas bei $13^{\circ}$ und $745 \mathrm{~mm}$ Druck. 
136 Lindner, Ueber die beiden isomeren Benzylderivate

\begin{tabular}{lcccc}
\multicolumn{3}{c}{ Berechnet für } & \multicolumn{3}{c}{ Gefunden } \\
& $\mathrm{C}_{14} \mathrm{H}_{14} \mathrm{~N}_{2} \mathrm{O}_{2}$ & & & \\
$\mathrm{C}$ & 69,42 & 69,19 & 69,03 & - \\
$\mathrm{H}$ & $\mathbf{5 , 7 9}$ & 5,73 & 6,12 & - \\
$\mathrm{N}$ & $11,5 \mathrm{5} 7$ & - & - & 11,72
\end{tabular}

\section{Molekulargewichtsbestionmung.}

$\begin{array}{cccccc}\text { Eisessig } & \text { Substanz } & \begin{array}{c}\text { Procent- } \\ \text { gehalt }\end{array} & \text { Erniedrigung } & \text { Mol.-Gew. } & \begin{array}{c}\text { Mol,-Gew. } \\ \text { berechnet }\end{array} \\ \mathbf{2 3 , 0 9} & 0,1340 & 0, \mathbf{0} 8 & 0,090^{\circ} & \mathbf{2 5 1} & 242 \\ \mathbf{2 3 , 0 9} & \mathbf{0 , 2 3 3 2} & \mathbf{1 , 0 1} & \mathbf{0 , 1 6 2 ^ { \mathbf { 0 } }} & \mathbf{2 4 3} & \end{array}$

Die letzten Mutterlaugen hinterliessen beim Verdunsten in reichlicher Menge ein $\mathrm{Oel}$, aus dem sich bei längerem Stehen geringe Mengen einer in Nadeln krystallisirenden Substanz absetzten, welche durch Trocknen auf dem Thonteller und Umkrystallisiren aus heissem Alkohol rein erhalten wurde. Durch den bei $73-74^{\circ}$ liegenden Schmelzpunkt, Löslichkeitsverhältnisse, Nitrosoreaction, Unlöslichkeit in Alkali und Analyse wurde der Körper als identisch mit dem Nitroso- $\alpha$-dibenzylhydroxylamin erkannt.

$0,2076 \mathrm{~g}$ gaben $0,5137 \mathrm{CO}_{3}$ und $0,1070 \mathrm{H}_{2} \mathrm{O}$.

$0,0525 \mathrm{~g}, \quad 5,10 \mathrm{ccm}$ Stickgas bei $15^{\prime \prime}$ und $745 \mathrm{~mm}$ Druck.

$\begin{array}{ccr} & \text { Berechnet für } & \text { Gefunden } \\ & \mathrm{C}_{\mathbf{1 4}} \mathrm{H}_{14} \mathrm{~N}_{\mathbf{2}} \mathrm{O}_{z} & \\ \mathrm{C} & 69,42 & 69,12 \\ \mathrm{H} & 5,79 & 5,86 \\ \mathrm{~N} & 1,1,57 & 11,33\end{array}$

Die Versuche, das bei $74^{0}$ schmelzende Nitrosamin durch Einwirkung von Benzylchlorid auf das Natrium- oder Silbersalz des Nitroso- $\beta$-benzylhydroxylamins in ätherischer oder benzolischer Suspension oder ohne Verdünnungsmittel in besserer Ausbeute za gewinnen, schlugen fehl.

Aus dem $\alpha$-Dibenzylhydroxylamin erhält man das bei $74^{\circ}$ schmelzende Nitrosamin in nahezu der berechneten Menge, wenn man zu einer warmen alkoholischen Lösung des salzsauren Salzes der Base die entsprechende Menge Natriumnitrit, ebenfalls in Alkohol gelöst, hinzubringt. Neben Chlornatrium 
scheidet sich beim Erkalten ein Theil des Nitrosamins ab, der Rest beim Verdünnen mit Wasser. Durch Umkrystallisiren aus heissem Alkohol oder Fällen der ätherischen Lösung mit Petroläther gewinnt man den Körper in weissen, brüchigen Nädelchen, die bei $73-74^{\circ}$ schmelzen. In Alkalien ist die Verbindung unlöslich, sie giebt sehr schön die Nitrosoreaction.

$0,1357 \mathrm{~g}$ gaben $0,3445 \mathrm{CO}_{2}$ und $0,0714 \mathrm{H}_{3} \mathrm{O}$.

$0,2065 \mathrm{~g} \quad, \quad 0,5255 \mathrm{CO}_{3}, 0,1095 \mathrm{H}_{2} \mathrm{O}$.

$0,1940 \mathrm{~g}, 18,9 \mathrm{ccm}$ Stickgas bei $13^{\circ}$ und $762 \mathrm{~mm}$ Druck.

\begin{tabular}{ccccc} 
& Berechnet für & \multicolumn{3}{c}{ Gefunden } \\
& $\mathrm{C}_{14} \mathrm{H}_{14} \mathrm{~N}_{2} \mathrm{O}_{2}$ & & & \\
$\mathrm{C}$ & 69,42 & 69,24 & 69,40 & - \\
$\mathrm{H}$ & 5,79 & 5,84 & 5,89 & - \\
$\mathrm{N}$ & 11,57 & - & - & 11,51
\end{tabular}

\section{Molekulargewichtsbestimmung.}

$\begin{array}{cccccc}\text { Eisessig } & \text { Substanz } & \begin{array}{c}\text { Procent- } \\ \text { gehalt }\end{array} & \text { Erniedrigung } & \text { Mol.-Gew. } & \begin{array}{c}\text { Mol.Gew. } \\ \text { berechnet }\end{array} \\ 19,51 & 0,1753 & 0,90 & 0.144^{\circ} & 243 & 242 \\ 19,51 & 0,4350 & 2,23 & 0.352^{\circ} & 247 & \end{array}$

Die Versuche, einen der beiden isomeren Körper in den anderen umzuwandeln, hatten keinen Erfolg.

Durch Kochen mit Alkohol werden die Nadeln nicht verändert; fügt man $z u$ der Lösung eine geringe Menge Natriumäthylat (ein Molekül $\mathrm{NaOC}_{2} \mathrm{H}_{5}$ auf vier Moleküle Nitrosamin) und kocht etwa dreiviertel Stunden, so erhält man beim Verdünnen ein röthliches $\mathrm{Oel}$, das nicht zum Krystallisiren zu bringen ist.

Bei gleicher Behandlung der Blättchen bildet sich eine geringe Menge Harz; man erhält fast die ganze angewandte Substanz unverändert zurück.

Da das Nitroso- $\beta$-benzylhydroxylamin sich beim Erwärmen seiner Lösung in Eisessig in das den hier besprochenen Verbindungen isomere Bisnitrosylbenzyl ${ }^{5}$ ) umwandelt, so erschien

3) Diese Annalen $\mathbf{2 6 3}, 221$. 
es nicht unmöglich, dass der bei $59^{\circ}$ schmelzende Körper seine Entstehung einer ähnlichen Umwandlung durch Natriumäthylat ohne Mitwirkung des Benzylchlorids verdanke. Der Versuch zeigte jedoch, dass dem nicht so ist. Als das Natriumsalz des Nitroso- $\beta$-benzylhydroxylamins zwei Stunden mit Alkohol gekocht wurde, kamen beim Ansăuern der mit Wasser verdünnten Lösung nur die bei $77-78^{\circ}$ schmelzenden, durch ihr chemisches Verhalten leicht erkennbaren Nadeln des Nitroso- $\boldsymbol{\beta}$-benzylhydroxylamins wieder zum Vorschein.

\section{Verhalten der Körper gegen Salzsäure.}

Die bei $74^{\circ}$ schmelzenden Nadeln wurden schon beim Einleiten von trocknem Chlorwasserstoff in ihre ätherische Lösung zersetzt. Nach einiger Zeit scheidet sich salzsaures $a$-Dibenzylhydroxylamin krystallinisch ab. Das Salz krystallisirte aus heissem Alkohol in breiten glänzenden Nadeln und schmolz unscharf bei $160-170^{\circ}$ unter Zersetzung.

$0,3244 \mathrm{~g}$ gaben $0,1867 \mathrm{AgCl}$.

$\begin{array}{ccc} & \text { Berechnet für } & \text { Gefunden } \\ & \mathrm{C}_{34} \mathrm{H}_{15} \mathrm{NO} . \mathrm{HCl} & \\ \mathrm{Cl} & 14,23 & 14,23\end{array}$

Alkalien machten die ölige Basis frei, welche durch Ueberführung in die bei $65-66^{\circ}$ schmelzende Benzoylverbindung ${ }^{4}$ ) identificirt wurde. Der Verbleib der Nitrosogruppe wurde nicht ermittelt. Vermuthlich bildet sich zunächst Nitrosylchlorid, welches einen Theil der Substanz in die öligen Nebenproducte überführt, die sich im Filtrate vom salzsauren $\alpha$-Dibenzylhydroxylamin vorfinden:

$$
\mathrm{C}_{7} \mathrm{H}_{7} \mathrm{~N}(\mathrm{NO}) \mathrm{OC}_{7} \mathrm{H}_{7}+2 \mathrm{HCl}=\mathrm{C}_{7} \mathrm{H}_{7} \mathrm{NHOC}_{7} \mathrm{H}_{7} \cdot \mathrm{HCl}+\mathrm{NOCl} \text {. }
$$

Im Gegensatz zu den Nadeln sind die Blättchen gegen Chlorwasserstoff in ätherischer Lösung beständig. Bei einstündigem Kochen mit concontrirter Salzsäure tritt zwar Geruch nach Benzylchlorid auf, doch wird auch hier der grösste Theil des Materials unverändert zurtackgewonnen.

4) Diese Annalen 257, 209. 
Erst bei andauerndem Erhitzen mit concentrirter Salzsäure auf $120-130^{\circ}$ findet völlige Spaltung unter Bildung von Benzylchlorid, Stickstoffoxyden und $\beta$-Benzylhydroxylamin neben verharzten Producten statt.

$3 \mathrm{~g}$ der Blättchen wurden mit überschüssiger 30 procentiger Salzsăure acht Stunden auf $120-130^{\circ}$ erhitzt. Das Rohr öffnete sich unter Druck, während sich Geruch nach Benzylchlorid und Stickstoffoxyden bemerklich machte. Ueber der salzsauren Lösung hatten sich $2,4 \mathrm{~g}$ Oel abgeschieden. Bei der Destillation desselben gingen bei $175-176^{\circ} 1,2 \mathrm{~g}$ einer wasserhellen Flüssigkeit von den Eigenschaften des Benzylchlorids über, der Rückstand zersetzte sich bei $180^{\circ}$.

Die salzsaure Lösung wirkte stark reducirend auf Fehlingsche Lösung. Nach dem Eindampfen hinterblieb ein theilweise verschmierter Rückstand, der neben anderen Producten salzsaures $\beta$-Benzylhydroxylamin enthielt. Durch Condensation mit Paranitrobenzaldehyd konnte daraus das bei $118^{\circ}$ schmelzende Benzylisoparanitrobenzaldioxim ${ }^{5}$ ) gewonnen werden, welches seinerseits durch physikalische Eigenschaften, Spaltung mit Salzsäure und Ueberführung in die bei $93-94^{0}$ schmelzende Doppelverbindung mit Paranitrobenzylisobenzaldoxim ${ }^{6}$ ) identificirt wurde. Die heisse alkoholische Lösung der Doppelverbindung schied nach Zusatz einer Spur Salzsäure den charakteristischen voluminösen Niederschlag von Paranitrobenzylisoparanitrobenzaldoxim $a b^{7}$ ).

Das $\boldsymbol{\beta}$-Benzylhydroxylamin entsteht zwar immer in ziemlich geringer Menge, da jedenfalls ein grosser Theil dieser gegen Oxydationsmittel äusserst empfindlichen Substanz durch die gleichzeitig gebildete salpetrige Säure zerstört wird, doch konnte sein Auftreten in dem Product bei mehrfach wiederholten Versuchen stets sicher festgestellt wurden.

5) Diese Annalen 283, 198.

*) Dieso Annalen 203, 203.

$\left.{ }^{7}\right)$ Diese Annalen $\mathbf{2 6 5}, 241$. 
Es geht daher aus diesen Versuchen hervor, dass in del Blättchen eine Benzylgruppe an ein Stickstoffatom gebunden ist, welches seinerseits mit Sanerstoff in Verbindung steht, dass ferner eine zweite Benzylgruppe durch Vermittelung von Sauerstoff gebunden ist und dass endlich ein Stickstoffatom vorhanden ist, welches in Form von salpetriger Säure oder Stickoxyd abgespalten werden kann.

\section{Verhalten gegen Reductionsmittel.}

Die Nadeln werden durch Zinnchlorür in salzsaurer Lösung leicht zu $a$-Dibenzylhydroxylamin und Hydroxylamin oder Ammoniak reducirt:

$$
\mathrm{C}_{7} \mathrm{H}_{7} \mathrm{~N}(\mathrm{NO}) \mathrm{OC}_{7} \mathrm{H}_{7}+2 \mathrm{H}_{2}=\mathrm{C}_{7} \mathrm{H}_{7} \mathrm{NHOC}_{7} \mathrm{H}_{7}+\mathrm{NH}_{3} \mathrm{O} \text {. }
$$

Nach einigen Versuchen wurde folgendermassen verfahren.

$5 \mathrm{~g}$ der Nadeln wurden in $125 \mathrm{ccm}$ absolutem Alkohol gelöst und $18 \mathrm{~g}$ Zinnchlorür, gelöst in $45 \mathrm{ccm} 30$ procentiger Salzsäure, hinzugegeben. Nach 36 Stunden wurde mit Wasser verdünnt, mit Alkali übersättigt und ausgeäthert. Die ätherische Lösung wurde vorsichtig mit wenig concentrirter Salzsäure versetzt und der entstehende Niederschlag abfiltrirt. Im Aether blieben nur $0,3 \mathrm{~g}$ eines gelblichen Oeles, welches keine Nitrosoreaction mehr gab. Die Analyse des salzsauren Salzes deutete auf das Vorliegen von salzsaurem Dibenzylhydroxylamin hin.

I. $0,2016 \mathrm{~g}$ gaben $0,1181 \mathrm{AgCl}$.

II. $0,2014 \mathrm{~g} \quad, \quad 0,1169 \mathrm{AgCl}$.

\begin{tabular}{cccc} 
& Berechnet für & \multicolumn{2}{c}{ Gefunden } \\
$\mathrm{Cl}$ & $\mathrm{C}_{\mathbf{1 4}} \mathrm{H}_{15} \mathrm{NO} . \mathrm{HCl}$ & $\mathrm{I}$. & II. \\
14,23 & 14,48 & 14,35
\end{tabular}

Durch Krystallisation aus heissem Alkohol wurde das Salz in breiten, glänzenden, ziemlich schwer löslichen Nadeln erhalten, die sich gegen $170^{\circ}$ allmählich zersetzten. $\mathrm{Zu}$ Analyse I diente das rohe Salz, zu Analyse II das aus Alkohol krystallisirte. Die durch Soda abgeschiedene ölige Base lieferte das bei $65-66^{\circ}$ schmelzende Benzoylderivat des $\alpha$-Dibenzylhydroxylamins. 
Die bei der Reduction erhaltene, von Dibenzylhydroxylamin befreite alkalische Lösung reducirte Fehling'sche Lösung und gab nach Zusatz von Benzaldehyd und Sättigen mit Kohlensäure an Aether Benzaldoxim $a b$, dass dem Aether durch Natronlauge entzogen und durch Ueberführung in $\beta$-Benzaldoxim identificirt wurde. Als bei einem anderen Versuche das Reductionsmittel mehrere Tage eingewirkt hatte, konnte an Stelle des Hydroxylamins nur Ammoniak nachgewiesen werden.

Durch Jodwasserstoff und Phosphor werden die Nadeln in Benzylamin, Stickoxyd and wahrscheinlich Benzyljodür übergeführt.

Die Nadeln wurden mit vier Gewichtstheilen $ّ 2$ procentiger Jodwasserstoffsäure und $1 / 2$ Gewichtstheil rothen Phosphors übergossen, während ein Kohlensäurestrom durch das Gefäss geleitet wurde. Die Reaction trat alsbald unter freiwilliger Erwärmung und Entwicklung von Gasen, die sich an der Luft roth färbten, ein. Nach Verlauf der ersten Einwirkung wurde noch kurze Zeit zum ruhigen Sieden erhitzt. Im Kühler zeigten sich reichlich Oeltropfen von heftigem Geruch nach Benzyljodür. Der Kolbeninhalt erstarrte beim Erkalten zu einem Brei von jodwasserstoffsaurem Benzylamin. Beim Behandeln mit Wasser und Aether nahm letzterer ein dickflüssiges oel auf, das zwar sehr heftig nach Benzyljodür roch, aus welchem dasselbe aber nicht in reinem Zustande gewonnen werden konnte, da es alsbald völlig verharzte.

Die wässrige Lösung wurde mit Alkali übersättigt und mit Dampf destillirt. Das aus dem Destillat gewonnene salzsaure Salz war in Wasser leicht löslich, schmolz bei 252-2530, lieferte eine in Wasser lösliche, durch Kali abscheidbare ölige Base, die an der Luft alsbald zu krystallinischem Carbonat erstarrte, und zeigte den für salzsaures Benzylamin berechneten Chlorgehalt.

$0,1650 \mathrm{~g}$ gahen $0,1662 \mathrm{AgCl}$.

Berechnet Gefunden

$\mathrm{Cl}$ 24,74 24,91 
Der mit Hülfe von Kaliumcyanat dargestellte Harnstoff schmolz bei $146^{\circ}$.

Ein Versuch, das Nitroso- $\alpha$-dibenzylhydroxylamin durch Zink und Eisessig in alkoholischer Lösung zu dem entsprechenden Hydrazin zu reduciren, gelang nicht.

Die Blättchen werden durch Zinnchlorür und Salzsäure in alkoholischer Lösung nur sehr schwierig angegriffen. Zwar zeigt die Lösung allmählich Reductionsvermögen gegen Fe bling' sche Lösung, doch gewinnt man selbst nach wochenlanger Einwirkung des Reductionsmittels fast das ganze Ausgangsmaterial unverändert zurück.

Ebenso wirkt Natriumamalgam auf die alkoholische Lösung so gut wie gar nicht ein; ja selbst als zu einer siedenden Lösung von $1 \mathrm{~g}$ der Substanz in $30 \mathrm{~g}$ absolutem Alkohol $1 \mathrm{~g}$ Natrium in mehreren Portionen hinzugefügt wurde, konnte weder Ammoniak noch ein anderes Reactionsproduct, sondern nur die unveränderten Blättchen nachgewiesen werden.

Dagegen verlief die Einwirkung von Jodwasserstoff und Phosphor in derselben Weise und unter Bildung derselben Producte, wie es oben bei der Reduction der Nadeln beschrieben ist. Nur trat die Reaction nicht freiwillig sondern erst nach kurzem Kochen ein. Das Benzyljodür konnte auch hier nicht in reinem Zustande gewonnen werden, sondern verharzte vollständig.

Das Benzylamin wurde durch die physikalischen Eigenschaften seines salzsauren Salzes, durch die Fähigkeit der Base, an der Luft zu Carbonat zu erstarren und durch Ueberführung in den bei $147^{\circ}$ schmelzenden Harnstoff identificirt. Analysirt wurde das bei $211-212^{\circ}$ unter Zersetzung schmelzende Platindoppelsalz.

$0,2130 \mathrm{~g}$ gaben $0,2122 \mathrm{CO}_{\mathrm{a}}$ und $0,0616 \mathrm{H}_{2} \mathrm{O}$.

$0,1521 \mathrm{~g}, 0,0473 \mathrm{Pt}$. 


$\begin{array}{lcr} & \text { Berechnet für } & \text { Gefunden } \\ & \mathrm{C}_{14} \mathrm{H}_{\mathbf{9 0}} \mathrm{N}_{2} \mathrm{PtCl}_{8} & \\ \mathrm{C} & 26,97 & 27,17 \\ \mathrm{H} & 3,21 & 3,21 \\ \mathrm{Pt} & 31,14 & 31,10\end{array}$

Die Unlöslichkeit der Blättchen in Alkalien macht es nicht gerade wabrscheinlich, dass die Verbindung eine Hydroxyloder Imidgruppe enthält. Zu demselben Schlusse führt auch das Verhalten gegen Benzoylchlorid und Phenylisocyanat, welche beide ohne Einwirkung auf den Körper sind. Essigsäureanhydrid bewirkt bei Gegenwart von Chlorzink tiefer gehende Zersetzung.

Von den beiden bei der Benzylirung des Nitroso- $\beta$-benzylhydroxylamins entstehenden Isomeren müssen die bei $74^{0}$ schmelzenden Nadeln auf Grund ibres chemischen Verhaltens sowie ihrer Entstehung aus $\alpha$-Dibenzyllydroxylamin als das Nitrosamin des letzteren Körpers aufgefasst werden.

Die bei $59^{\circ}$ schmelzenden Blättchen dürften, obgleich sie ebenso leicht wie die Nadeln die Nitrosoreaction geben und unter der Einwirkung der Salzsäure sowie von Reductionsmitteln wenn gleich viel schwieriger dieselben Producte liefern wie die Nadeln, nicht stereoisomer mit den letzteren sein, sondern eine andere Structur besitzen. Eine Beständigkeit, wie sie der Körper namentlich den Reductionsmitteln gegenüber aufweist, ist mit der Auffassung dessolben als Nitrosamin nicht vereinbar.

Für die Frage nach der Constitntion der Blăttchen sind folgende Thatsachen massgebend.

1) Die Verbindung enthält zwei Benzylgruppen, von denen die eine durch Vermittelung von Sauerstoff gebunden ist, da sie bei der Spaltung mit Salzsäure als Benzylchlorid, bei der Reduction mit Jodwasserstoff als Benzyljodid abgespalten wird. Die zweite Benzylgruppe ist an ein mit Sauerstoff beladenes Stickstoffatom gebunden, da sie bei der Spaltung mit Salzsäure 
144 Lindner, Ueber die beiden isomeren Benzylderivate etc.

als $\beta$-Benzylhydroxylamin, bei der Reduction mit Jodwasserstoff als Benzylamin austritt.

2) Ein Stickstoffatom tritt bei energischen Eingriffen in Form von Stickstoffoxyden aus.

3) Hydroxyl- oder Imidgruppen sind wahrscheinlich nicht vorhanden.

Diesen Thatsachen würden die folgenden Formeln Rechnung tragen :

$$
\text { I. } \mathrm{C}_{7} \mathrm{H}_{7}-\mathrm{N}=\mathrm{N}-\mathrm{OC}_{7} \mathrm{H}_{7} \quad \text { II. } \mathrm{C}_{7} \mathrm{H}_{7}-\mathrm{N}=\mathrm{NOC}_{7} \mathrm{H}_{7} .
$$

Für das Nitroso- $\beta$-benzylhydroxylamin ergiebt sich aus seiner Entstehung am wahrscheinlichsten die Formel $\mathrm{C}_{7} \mathrm{H}_{7} \mathrm{~N}(\mathrm{NO}) \mathrm{OH}$, und da nunmehr der Nachweis erbracht ist, dass bei Benzylirung des Körpers Nitroso- $\alpha$-dibenzylhydroxylamin entsteht, so ist kein Grund mehr vorhanden, an der Berechtigung dieser Auffassung zu zweifeln.

Um die Entstehung des zweiten Benzylderivates zu erklären, muss man annehmen, dass bei der Einwirkung von Natriumäthylat und Benzylchlorid das Nitroso - $\beta$-benzylhydroxylamin zum Theil umgelagert wird:

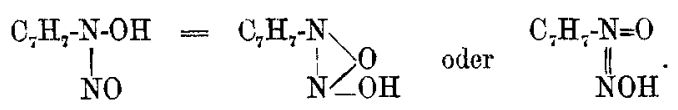

Dass eine Verbindung von der Constitution, wie sie hier für die bei $59^{\circ}$ schmelzenden Blättchen angenommen ist, so leicht und schön die Nitrosoreaction zeigt, kann nichts Auffälliges bieten, da nach Abspaltung der an Sauerstoff gebundenen Benzylgruppe durch die concentrirte Schwefelsäure Nitroso$\beta$-benzylhydroxylamin, zunächst in der tautomeren Form, regenerirt wird.

(Greschlossen am 20. April 1893.) 